\title{
An Adaptive Component Mode Synthesis Method for Dynamic Analysis of Jointed Structure with Contact Friction Interfaces
}

\author{
Jie Yuan ${ }^{\mathrm{a}, *}$, Loic Salles ${ }^{\mathrm{a}}$, Fadi El Haddad ${ }^{\mathrm{a}}$, Chian Wong $^{\mathrm{b}}$ \\ ${ }^{a}$ Vibration University Technology Centre, Imperial College London SW7 2AZ, London, UK \\ ${ }^{b}$ Rolls Royce Plc, DE24 8BJ, Derby, UK
}

\begin{abstract}
Component model synthesis (CMS) has been widely used for model order reduction in dynamic analysis of jointed structures with localized non-linearities. The main drawback of these CMS methods is that their computational efficiency largely depends on the size of contact friction interfaces. This work proposes an adaptive reduction approach to improve these CMS based reduction methods in the application to the assembled structure with frictional interfaces. The main idea of this method is that, instead of retaining the whole frictional interface DOFs in the reduced model, only those DOFs in a slipping or separating condition are retained. This would significantly reduce the size of classical CMS based reduced models for dynamical analysis of jointed structure with micro-slip motion, leading to an impressive computational saving. This novel approach is based on a reformulated dynamic system that consists of a underlying linearised system and including an updating internal variable to account the effects of non-linear contact friction force on the interface. The paper also describes the detailed implementation of the proposed approach with harmonic balanced method for non-linear spectral analysis, where a new updating algorithm is put forward to enable the size of the reduced model can be automatically updated according to the contact condition of interface nodes. Two distinct FE joint
\end{abstract}

\footnotetext{
* Corresponding author

Email address: jie.yuan@imperial.ac.uk (Jie Yuan)
}

Preprint submitted to Computers and Structures

September 20, 2019 
models are used to validate the proposed method. It is demonstrated that the new approach can achieve a considerable computing speed-up comparing to the classic CMS approach while maintain the same accuracy.

Keywords: Reduced order modelling, Component mode synthesis, Harmonic balanced methods, Jointed structures, Contact friction

\section{Introduction}

Mechanical joints have been widely present in both aerospace and civil structures, e.g. bolted joints in bridges to fir-tree and dovetail joint in aero-engines [1, 2]. They are represented as an effective and affordable way to hold together

5 the assembly and transmit the loadings between the components [3, 4]. However, due to the relative motions occurring on the jointed interfaces, the forced dynamic repose of the whole assembly can be substantially changed. This interfacial relative motion can introduce additional energy dissipation that would change the overall stiffness of an assembly and thus lead to a shift of resonance frequencies [5]. However, the amount of damping due to these joints is typically 10-100 times higher than normal material damping [6]. The experiments also consistently exhibit that the damping in these joints vary with the amplitude of excitation levels [5]. To guarantee the service life of these joints, it is therefore crucial for engineers to understand their modal properties. However, the cost involving manufacturing and testing these jointed structure are commonly high and sometimes are unaffordable in most cases. It is therefore important to have predictive models beforehand that can reliably estimate the dynamical response of such jointed structures. However, due to the strong nonlinearities from the contact friction in joints, linear or linearised predictive models are not accurate 20 enough to capture the nonlinear dynamics of these structures [6]. It is necessary to include intuitively constitutive models that provide a better description of joint mechanics in modelling these joints. In this way, nonlinear dynamic effects in the joints can be taken into account. To obtain the dynamical response for nonlinear joint models, this would require iterative solvers. Most existing mod- 
elling approaches including the non-linear contact friction models employ either time domain solvers [7] (e.g.Runge-Kutta or Newmark method [8]) or frequency domain solvers 6, 9] (e.g.harmonic balance methods (HBM)). To solve the full scale jointed model without reduction for a wide range of frequencies or time, the computational expense with these solvers is usually unacceptable. HBM is well known for its high efficiency to obtain the steady state approximations that can reduce the computational time by several orders compared to time domain methods [10]. However, even with the use of HBM, modelling the joints in high fidelity still suffers from the unaffordable computational cost and numerical stability issues [11]. Fortunately, ROM methods provide a viable solution to incorporate these accurate contact friction models into assembled models.

To date, the reduced model for the structure with joints are mostly constructed using substruturing methods in the class of CMS techniques [10, 9, 11, 12, 13. The main concept behind these CMS based methods consists in assuming two or more connected components in an assembly are distinct sub-

40 structural elements so that their modal bases can be separately calculated via deterministic FE analysis [14. The full system can be then significantly reduced via Galerkin Projection [12, 15] using the reduced basis that combines the linear normal modes from different components with the static impulse modes associated to retained interface nodes. This can effectively eliminate most in-

45 ternal DOFs. The CMS based reduced models would retain the whole DOFs involved in on the joint interface [16. The nonlinear contact friction model therefore can be conveniently integrated through these retained DOFs. Rubin and Craig-Bampton (CB) method are two typical methods based on CMS techniques [17, 18]. Rubin method combines free interface modes and static ones obtained from the static solution of applied interface load, which is also called attachment modes or flexible residual. In contrast, $\mathrm{CB}$ method combines the normal modes with the fixed interface condition and static solutions (so called constrain modes) obtained by applying unit displacement at each interface DOF. Both methods have been proven effective for ROM of the structures with frictional interfaces [9, 10. Unfortunately, the main drawbacks of these CMS based 
approaches is that their size heavily depends on the number of DOFs involved in contact interfaces [19, 12]. This limitation compromises the computational efficiency of CMS based ROMs especially in the case where the contact interface regions are intensive in order to ensure an accurate stress recovery for reliable fatigue life predictions. To improve these CMS based methods, two interface reduction methods were proposed to reduce static impulse modes. One of them is called common interface reduction approach that was proposed by Becker and Gaul [20, 21]. The main idea is to replace full size of constrain modes by a subset of interface modes, which are calculated via performing a second modal analysis related to interface DOFs. The other one is called joint interface modes approach proposed by Witteveen [19]. The joint interface modes are generated explicitly by respecting the Newtons third law across the joint interfaces. A comparative study by Becker 20] shows both methods can effectively reduce interface DOFs but joint interface modes can achieve better convergence with much less number of interface modes [20]. However, in general, the convergence of these two methods are problem dependent and heavily reply on a good selection of such special interface modes. In addition, the evaluation of these interface models would increase the off-line cost. For these two reasons, these two interface reduction methods still have not been widely used for the 75 simulations of jointed structures.

Instead of using the condensed interface modes, this work aims to develop an adaptive approach to further improve reduced models constructed by classical CMS based methods. The main idea of the proposed approach is to adaptively remove the fully stuck contact nodes from the classical ROMs depending on the contact condition on the joint interface, which would lead to a significant reduction in the number of static modes. This idea is inspired by the previous findings that, for the majority of structures with joints, the contact interfaces are mostly in a micro-slip motion where most of the contact nodes are in a stuck condition under the dynamic loads. This phenomenon has been widely found

85 in bolted joints structures [5] and fir-tree joints in turbines [22]. Fig[1] shows an example of forced frequency response of a turbine with root joints, and also 

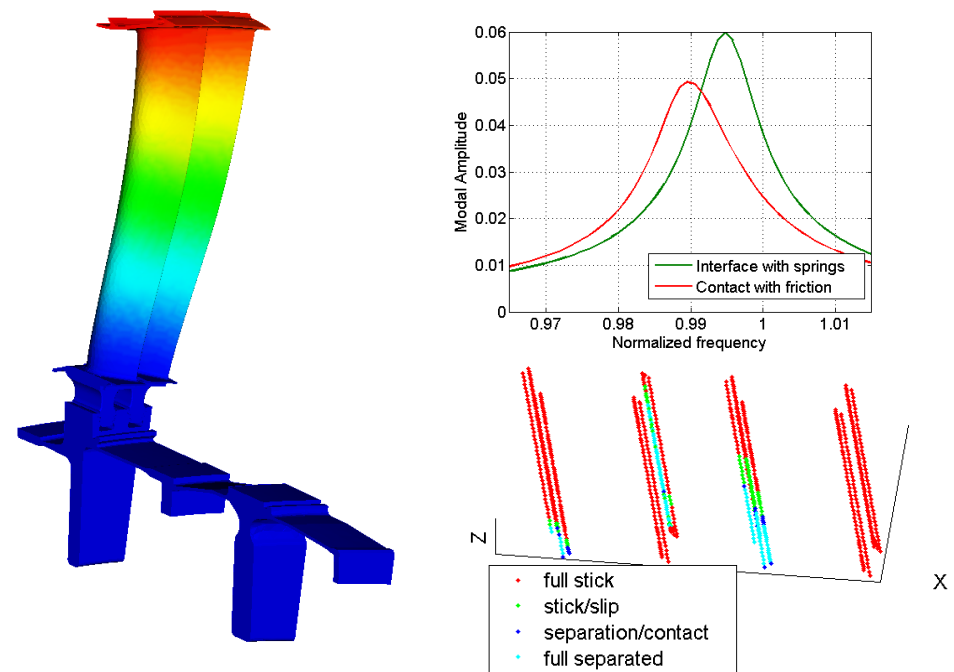

Figure 1: An example of the contact conditions of a turbine during the vibrations

the corresponding contact conditions in the root joint interface at the resonance frequency 22]. It can be clearly seen that, even at the resonance frequency, the majority of the contact nodes in the root joint are still in a sticking condition and only a small portion of contact nodes in a slipping or separation condition. The rationale behind this adaptive approach is that, instead of including whole interface DOFs into the reduced model, only include contact interface DOFs in a slipping and separating condition while the dynamics associated to those stuck nodes would be considered in the reformulated linearised system. In this way, the size of ROM for a jointed structure can be significantly reduced for jointed structures with micro-slip motion compared to the CMS based reduced models. The initial study presented in 22] found that such an adaptive approach can be achievable by reformulating original dynamic system using a linearised system that assuming all contact nodes in a sticking condition and an internal penalty variable that accounts the non-linear effects introduced by those nodes in a slipping or separating condition. However, the study has not yet addressed the challenge of how to enable such adaptive size update with state of the art HBM solvers. 
The objective of this paper is to present the entire mathematical derivations

\subsection{Equation of motion}

A dynamic system consisting of two connected components with contact interfaces is considered. Fig 2 shows an illustration of such a jointed structure with localized nonlinearities. Using the finite element discretisation, the ordinary differential governing equation describing the dynamics of this assembled system can be expressed as:

$$
\left[\begin{array}{cc}
\mathbf{M}^{\mathbf{1}} & \mathbf{0} \\
\mathbf{0} & \mathbf{M}^{\mathbf{2}}
\end{array}\right]\left[\begin{array}{l}
\ddot{u}^{1} \\
\ddot{u}^{2}
\end{array}\right]+\left[\begin{array}{cc}
\mathbf{K}^{\mathbf{1}} & \mathbf{0} \\
\mathbf{0} & \mathbf{K}^{\mathbf{2}}
\end{array}\right]\left[\begin{array}{l}
u^{1} \\
u^{2}
\end{array}\right]=\left[\begin{array}{c}
F_{e x}^{1} \\
F_{e x}^{2}
\end{array}\right]-\left(\left[\begin{array}{l}
\mathbf{B}^{\mathbf{1}} \\
\mathbf{B}^{\mathbf{2}}
\end{array}\right] F_{n l}\left(u_{b}^{1}, u_{b}^{2}\right)\right)
$$

It is assumed the amount of DOFs for each sub-structure is $N$ and the amount of contact friction DOFs for each contact interface is $M . \mathbf{M}^{\mathbf{1}}, \mathbf{M}^{\mathbf{2}}, \mathbf{K}^{\mathbf{1}}, \mathbf{K}^{\mathbf{2}}$ are the mass and stiffness matrix of two connected sub-structures and all of them 


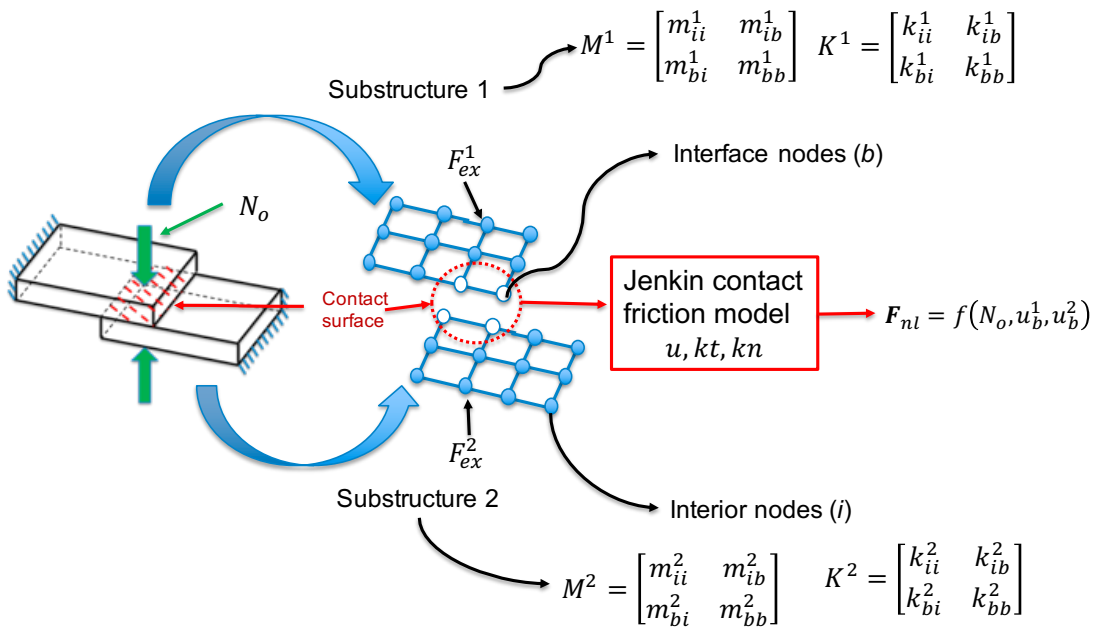

Figure 2: An illustration of 3D joint beams with contact friction non-linearities

125

have the same dimensions of $N \mathrm{x} N ; F_{e x}^{1}, F_{e x}^{2}$ is the harmonic external forced excitation on two substructures; $F_{n l}$ are the $M \mathrm{x} 1$ nonlinear contact friction force vector, which is as a function of the contact interface displacement $u_{b}^{1}, u_{b}^{2}$. $\mathbf{B}^{\mathbf{1}}, \mathbf{B}^{\mathbf{2}}$ are the $N \mathrm{x} M$ signed boolean matrix acting on the contact interface for each substructure.

\subsection{Contact friction modelling}

A node-to-node modelling approach, namely Jenkin model [6], is used to calculate the contact friction forces $F_{n l}$. The contact friction contains three internal states, namely stick/stuck, slip and gap. The occurrence of these states depends on the level of pre-loading applied on the interface and also the ampli-

135 tude of external force. Using this Jenkin model, the contact friction forces for $i^{t h}$ contact node pair can be formulated as follows:

$$
F_{n l, x}^{i}=\left\{\begin{array}{cc}
k_{t}\left(\Delta x^{i}-\Delta x_{c}^{i}\right)+N_{0}^{x, i} & \text { stick } \\
\mu F_{n l, z}^{i} \sin (\theta) & \text { slip } \\
0 & \text { gap }
\end{array}\right.
$$




$$
\begin{aligned}
& F_{n l, y}^{i}=\left\{\begin{array}{cc}
k_{t}\left(\Delta y^{i}-\Delta y_{c}^{i}\right)+N_{0}^{y, i} & \text { stick } \\
\mu F_{n l, z}^{i} \cos (\theta) & \text { slip } \\
0 & \text { gap }
\end{array}\right. \\
& F_{n l, z}^{i}=\left\{\begin{array}{cc}
k_{n} \Delta z^{i}+N_{0}^{z, i} & \text { stick or slip } \\
0 & \text { gap }
\end{array}\right.
\end{aligned}
$$

Where $\Delta x^{i}, \Delta y^{i}$ and $\Delta z^{i}$ are the relative displacement of $i^{t h}$ contact node pair in tangential (x,y) and normal (z) direction; $\Delta x_{c}^{i}, \Delta y_{c}^{i}$ are the internal state variables that represent the position of tangential sliders. They would become zero if the contact node were in a pure sticking condition; $N_{0}^{i, x}, N_{0}^{i, y}, N_{0}^{i, z}$ is tangential (x, y) and normal (z) components of interface pre-loading $N_{0}$ illustrated in Fig $2 \mu$ is the friction coefficient of the contact interface; $F_{n l, x}^{i}, F_{n l, y}^{i}$ are the tangential friction force and $F_{n l, z}^{i}$ is the normal contact force; $\theta$ is the angle to decompose the tangential friction force in $\mathrm{x}$ and $\mathrm{y}$ direction. It is determined by the predicted tangential force as:

$$
\tan (\theta)=\frac{k_{t}\left(\Delta x^{i}-\Delta x_{c}^{i}\right)+N_{0}^{x, i}}{k_{t}\left(\Delta y^{i}-\Delta y_{c}^{i}\right)+N_{0}^{y, i}}
$$

The stick condition occurs when the predicted tangential force is less than the critical slipping force, namely $\sqrt{\left(F_{n l, x}^{i}\right)^{2}+\left(F_{n l, y}^{i}\right)^{2}} \leq \mu F_{n l, z}^{i}$. The contact force would vary proportional to relative displacement and there would be no energy dissipation on the contact interface. Otherwise, the slip condition would occur and the amplitude of tangential frictional force would be $\mu F_{n l, z}^{i}$. The energy dissipation would happen then. The gapping condition would happen when predicted normal force $F_{n l, z}^{i}$ were less than zero. In this case, the contact pair is separated and all the tangential and normal force would become zero.

With this contact friction model, $F_{n l}$ shown in Eq. 1 can be evaluated and assembled for all the contact pairs. Assuming that local coordinate systems for all the contact nodes are consistent with the global system defined for the FE 
model, $F_{n l}$ for $m$ contact pairs can be written as:

$$
F_{n l}=\left[\begin{array}{lllllll}
F_{n l, x}^{1} & F_{n l, y}^{1} & F_{n l, z}^{1} & \cdots & F_{n l, x}^{m} & F_{n l, y}^{m} & F_{n l, z}^{m}
\end{array}\right]^{T}
$$

\section{Benchmark Rubin method}

This section is to give a brief review of the reduced basis of CMS based Rubin method, which would be used to benchmark the results from new ROM in this study. As mentioned in the introduction, Rubin method combines free interface normal modes and static modes (or called attachment modes) as its reduced basis 23 . The free interface modes are calculated for each component as:

$$
-\omega^{2}\left[\mathbf{M}^{(i)}\right]\left[\phi^{(i)}\right]+\left[\mathbf{K}^{(i)}\right]\left[\phi^{(i)}\right]=0
$$

Where $\phi^{(i)}$ are the dynamical modes for $i^{\text {th }}$ substructure. The static modes that used to account the interface effects in the high frequency response are evaluated by solving the static problem of each substructure under applied unit force on the interface. They are generally the inverse or the generalized inverse of stiffness matrix if rigid body motions are existing. By eliminating the redundant information included in the dynamical modes, the static modes $\mathbf{G}^{(i)}$ for $i^{\text {th }}$ component can be expressed as:

$$
\mathbf{G}^{(i)}=\mathbf{K}^{(i)+}-\sum_{j=1}^{n_{\theta}} \frac{\phi_{j}^{(i)} \phi_{j}^{(i)^{T}}}{\omega_{j}^{(i)^{2}}}
$$

Where $\mathbf{K}^{(i)+}$ the generalized inverse of stiffness matrix; $n_{\theta}$ is the number of

dynamic modes; $\omega_{j}^{(i)}$ is the the natural frequency to $j^{t} h$ mode for $i^{t h}$ component. The transformation equation without the presence of rigid modes can be expressed:

$$
\left[\begin{array}{l}
u^{1} \\
u^{2}
\end{array}\right]=\underbrace{\left[\begin{array}{cccc}
\phi^{(1)} & -\mathbf{G}^{(1)} \mathbf{B}^{(1)} & 0 & 0 \\
0 & 0 & \phi^{(2)} & -\mathbf{G}^{(2)} \mathbf{B}^{(2)}
\end{array}\right]}_{\mathbf{\Phi}_{\mathbf{R B}}}\left[\begin{array}{c}
\eta^{(1)} \\
\lambda^{(1)} \\
\eta^{(2)} \\
\lambda^{(2)}
\end{array}\right]
$$


Where $\boldsymbol{\Phi}_{\mathbf{R B}}$ is the transformation matrix for the Rubin method; $\eta^{(1)}, \eta^{(2)}$ are

the modal participation factor for two substructures; $\lambda^{(1)}, \lambda^{(2)}$ are interface force for both substructures. The size of reduced basis is proportional to the size of contact interface DOFs

\section{Adaptive CMS method}

\subsection{Linearised dynamic system}

The proposed ROM method is formulated based on the underlying linearised system of jointed structures where the joint is linearised by assuming all of the contact nodes are in a pure stick condition. In this way, the sticking contact forces for each contact node $i$ can be expressed as a linear function of relative displacement on the interface as:

$$
F_{n l, s t i c k}^{i}=\left[\begin{array}{l}
F_{n l, x, s t i c k}^{i} \\
F_{n l, y, s t i c k}^{i} \\
F_{n l, z, s t i c k}^{i}
\end{array}\right]=\underbrace{\left[\begin{array}{ccc}
k t & 0 & 0 \\
0 & k t & 0 \\
0 & 0 & k n
\end{array}\right]}_{\mathbf{K}_{\mathbf{J o i n t}}^{\mathbf{L}, \mathbf{i}}}\left[\begin{array}{c}
\Delta x^{i} \\
\Delta y^{i} \\
\Delta z^{i}
\end{array}\right]
$$

Where $\mathbf{K}_{\text {Joint }}^{\mathbf{L}, \mathbf{i}}$ is local joint stiffness matrix for $i^{\text {th }}$ contact node. It is worth noting that the static pre-loading $N_{0}^{x, i}, N_{0}^{y, i}, N_{0}^{z, i}$ are not included because they would not affect the dynamic response of joint structures. Since the dynamical analysis is performed based on the static equilibrium position, the pre-loading $N_{0}$ distribution obtained from quasi-static analysis would not be included in the dynamical formulation. In this way, the linear sticking contact force for all $m$ contact nodes can be then evaluated. The corresponding local joint stiffness matrix can be expressed as:

$$
\mathbf{K}_{\text {joint }}^{\mathrm{L}}=\operatorname{Diag}\left(\mathbf{K}_{\text {joint }}^{\mathbf{L}, \mathbf{1}}, \mathbf{K}_{\text {joint }}^{\mathbf{L}, \mathbf{2}}, \cdots, \mathbf{K}_{\text {joint }}^{\mathbf{L}, \mathbf{m}}\right)
$$

Where $\mathbf{K}_{\text {joint }}^{\mathbf{L}}$ is the $M \mathrm{x} M$ diagonal joint contact stiffness matrix. To integrate it with global unknown vectors, the local joint stiffness matrix can be transformed 
using the Boolbean matrix as:

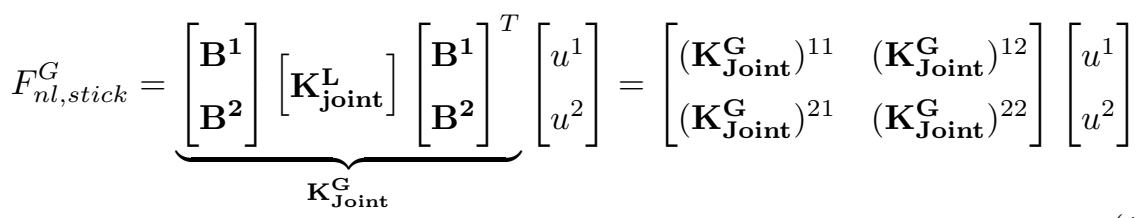

Where $F_{n l, s t i c k}^{G}$ is a $2 N \times 1$ global contact force vector; $\mathbf{K}_{\mathbf{J} \text { oint }}^{\mathrm{G}}$ is the transformed $2 N \times 2 N$ global joint stiffness matrix. To substitute the non-linear contact friction in Eq1 with Eq 12 , the linearised dynamic system can be expressed as:

$$
\underbrace{\left[\begin{array}{cc}
\mathbf{M}^{\mathbf{1}} & \mathbf{0} \\
\mathbf{0} & \mathbf{M}^{2}
\end{array}\right]}_{\mathbf{M}}\left[\begin{array}{c}
\ddot{u}^{1} \\
\ddot{u}^{2}
\end{array}\right]+\underbrace{\left[\begin{array}{cc}
\mathbf{K}^{1}+\left(\mathbf{K}_{\mathbf{J o i n t}}^{\mathbf{G}}\right)^{11} & \left(\mathbf{K}_{\mathbf{J o i n t}}^{\mathbf{G}}\right)^{12} \\
\left(\mathbf{K}_{\mathbf{J o i n t}}^{\mathbf{G}}\right)^{21} & \mathbf{K}^{\mathbf{2}}+\left(\mathbf{K}_{\mathbf{J o i n t}}^{\mathbf{G}}\right)^{22}
\end{array}\right]}_{\mathbf{K}_{\text {linearised }}}\left[\begin{array}{c}
u^{1} \\
u^{2}
\end{array}\right]=\left[\begin{array}{c}
F_{\text {ex }}^{1} \\
F_{\text {ex }}^{2}
\end{array}\right]
$$

165 matrix.

\subsection{Internal Penalty variable}

The dynamic response from the linearised system in Eq. 15 would not be accurate if there are some contact nodes in either slipping or gapping conditions. It is because these contact states would introduce the nonlinear contact friction force into the system which can not be simply expressed as a linear function of relative displacement on the interface. An internal penalty variable $\Delta p$ is therefore introduced here to account those additional nonlinear dynamical effects from the joint interface.

The internal penalty variable is defined as the static response in joint interface by applying the residual force between the actual non-linear contact force and the linear contact force assuming when all the contact nodes in a stick 
condition. It can be formulated as follows:

$$
\begin{aligned}
& \Delta p=\left[\left(\mathbf{K}_{\text {joint }}^{\mathbf{L}}\right)^{-1}\right]\left(F_{n l}-F_{n l, s t i c k}\right) \\
& =\left[\left(\mathbf{K}_{\text {joint }}^{\mathbf{L}}\right)^{-1}\right](F_{n l}-\mathbf{K}_{\text {joint }}^{\mathbf{L}} \underbrace{\left[\begin{array}{c}
\mathbf{B}^{\mathbf{1}} \\
\mathbf{B}^{\mathbf{2}}
\end{array}\right]^{T}\left[\begin{array}{l}
u^{1} \\
u^{2}
\end{array}\right]}_{\Delta u_{b}})
\end{aligned}
$$

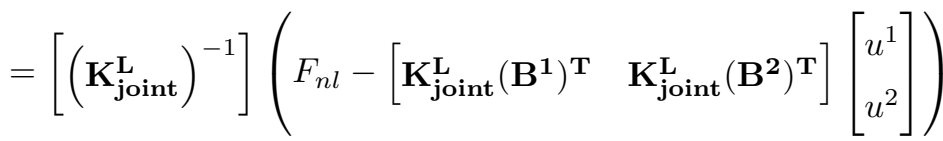

175 is an assembly of $\Delta x, \Delta y, \Delta z$ for all the contact pairs. The dimension of full $\Delta p$ is $M \mathrm{x} 1$. If any contact node were in a stick condition, the corresponding part in $\Delta p$ would be zero.

\subsection{Reformulated dynamic system} penalty variable Eq 14 into the linearised dynamic system in Eq. 13 . The new dynamic system for joint structure can be expressed as:

$$
\underbrace{\left[\begin{array}{ccc}
\mathbf{M}^{1} & \mathbf{0} & \mathbf{0} \\
\mathbf{0} & \mathbf{M}^{2} & \mathbf{0} \\
\mathbf{0} & \mathbf{0} & \mathbf{0}
\end{array}\right]}_{\mathbf{M}_{\text {New }}}\left[\begin{array}{c}
\ddot{u}^{1} \\
\ddot{u}^{2} \\
\Delta \ddot{p}
\end{array}\right]+\underbrace{\left[\begin{array}{ccc}
\mathbf{K}^{1}+\left(\mathbf{K}_{\text {Joint }}^{\mathbf{G}}\right)^{11} & \left(\mathbf{K}_{\text {Joint }}^{\mathbf{G}}\right)^{12} & \mathbf{B}^{1} \mathbf{K}_{\text {Joint }}^{\mathbf{L}} \\
\left(\mathbf{K}_{\text {Joint }}^{\mathbf{G}}\right)^{21} & \mathbf{K}^{2}+\left(\mathbf{K}_{\text {Joint }}^{\mathbf{G}}\right)^{22} & \mathbf{B}^{2} \mathbf{K}_{\text {Joint }}^{\mathbf{L}} \\
\mathbf{K}_{\text {Joint }}^{\mathbf{L}}\left(\mathbf{B}^{1}\right)^{\mathbf{T}} & \mathbf{K}_{\text {Joint }}^{\mathbf{L}}\left(\mathbf{B}^{2}\right)^{\mathbf{T}} & \mathbf{K}_{\text {Joint }}^{\mathbf{L}}
\end{array}\right]}_{\mathbf{K}_{\text {New }}}\left[\begin{array}{c}
u^{1} \\
u^{2} \\
\Delta p
\end{array}\right]=\left[\begin{array}{c}
F_{e x}^{1} \\
F_{e x}^{2} \\
F_{n l}
\end{array}\right]
$$

Where $\mathbf{M}_{\mathbf{N e w}}, \mathbf{K}_{\mathrm{New}}$ are the global mass and stiffness matrix for new dynamic system. This new dynamic system is completely equivalent to the original dynamic system in Eq1. The main advantage of this formulation is that the zero part of of $\Delta p$ can be now removed from system without any change in the dynamics of the system. In the case of full sticking condition, the whole $\Delta p$ would be zero and can be wholly removed from Eq, 15 . This feature is critical for the development of the adaptive reduced order model in the following section. 
This section describes the development of an adaptive ROM from the reformulated dynamic system in Eq,15 Similar to classical CMS method, the reduced basis for the proposed approach also includes two types of reduced basis, namely the linear vibrational modes and constrain (static) modes related to the interface DOFs. For the proposed method, the linear normal modes would be obtained by solving the eigenvalue problem of the linearised system in Eq 13

$$
-\omega^{2}[\mathbf{M}][\phi]+\left[\mathbf{K}_{\text {linearised }}\right][\phi]=0
$$

Where $\phi$ are the modes of the linearised system. The static modes are obtained by applying unit displacement vectors on the DOFs related to internal penalty variable as follows:

$$
\left[\mathbf{K}_{\text {New }}\right]\left[\begin{array}{l}
\psi \\
\mathbf{I}
\end{array}\right]=\left[\begin{array}{l}
\mathbf{0} \\
\mathbf{R}
\end{array}\right],[\psi]=\left[\mathbf{K}_{\text {linearised }^{-1}}\right]\left[\begin{array}{l}
\mathbf{B}^{1} \mathbf{K}_{\mathbf{J} \text { oint }}^{\mathbf{L}} \\
\mathbf{B}^{2} \mathbf{K}_{\mathbf{J} \text { oint }}^{\mathbf{L}}
\end{array}\right]
$$

Where $\mathbf{I}$ is the $M \times M$ identity matrix; $\psi$ is the full set of constrain modes with a size of $2 N \mathrm{x} M ; \mathbf{R}$ are the $M \mathrm{x} M$ reaction force matrix. The transformation of the unknowns from physical domain to the modal domain can be expressed as:

$$
\left[\begin{array}{c}
u^{1} \\
u^{2} \\
\Delta p
\end{array}\right]=\left[\begin{array}{cc}
\phi & \psi \\
\mathbf{0} & \mathbf{I}
\end{array}\right]\left[\begin{array}{c}
\eta \\
\Delta p
\end{array}\right]=\underbrace{\left[\begin{array}{ll}
\phi & \psi \\
\mathbf{0} & \mathbf{I}
\end{array}\right]\left[\begin{array}{cc}
\mathbf{I} & \mathbf{0} \\
\mathbf{0} & \mathbf{B}
\end{array}\right]}_{\mathbf{\Phi}}\left[\begin{array}{c}
\eta \\
\Delta p_{R}
\end{array}\right],[\mathbf{\Phi}]=\left[\begin{array}{cc}
\phi & \psi \mathbf{B} \\
\mathbf{0} & \mathbf{B}
\end{array}\right]
$$

Where $\eta$ is the modal participation factors of the selected dynamic modes; $\Delta p_{R}$ is the non-zero part of $\Delta p ; \mathbf{B}$ is the Boolean matrix that abstract on the nonzero part of $\Delta p ; \boldsymbol{\Phi}$ is the transformation matrix whose size depends on the size of non-zero part of $\Delta p$. Such an adaptive reduced basis can significantly reduce the size of the reduced model for jointed structures if most of contact nodes are in a stick condition during the vibration e.g. jointed structures. Using the Galerkin projection with the new reduced basis, the new reduced dynamic system can be expressed as:

$$
\left[\mathbf{M}_{\mathbf{R}}\right][\ddot{q}(t)]+\left[\mathbf{K}_{\mathbf{R}}\right][q(t)]=\left[\begin{array}{c}
F_{e x}^{R} \\
F_{n l}^{R}
\end{array}\right]
$$




$$
\begin{gathered}
\mathbf{M}_{\mathbf{R}}=\boldsymbol{\Phi}^{T} \mathbf{M}_{\mathbf{N e w}} \boldsymbol{\Phi}, \mathbf{K}_{\mathbf{R}}=\boldsymbol{\Phi}^{T} \mathbf{K}_{\mathbf{N e w}} \boldsymbol{\Phi} \\
q=\left[\begin{array}{c}
\eta \\
\Delta p_{R}
\end{array}\right], F_{e x}^{R}=\boldsymbol{\Phi}^{T} F_{e x}, F_{n l}^{R}=\boldsymbol{\Phi}^{T} F_{n l}(\boldsymbol{\Phi} q(t))
\end{gathered}
$$

$\mathbf{K}_{\mathbf{R}}$ and $\mathbf{M}_{\mathbf{R}}$ are the reduced mass and stiffness matrix of the new dynamic system; $q$ is the reduced unknown for the dynamic system; $F_{e x}^{R}$ and $F_{n l}^{R}$ are the reduced external modal force and non-linear force vector.

\section{HBM with automatic size updating}

This section is to describe how to integrate the developed adaptive reduced order model with the harmonic balanced method for spectral analysis of nonlinear jointed system. The basic of harmonic balanced method would be briefly introduced at first followed by the presentation of a novel updating algorithm for achieving the automatic size updating with the proposed adaptive ROM.

\subsection{Harmonic balanced method}

The idea of the multi-harmonic balanced method is to approximate the steady state response of the system by using a truncated Fourier series. The main advantage of this HBM is that it allows much faster computations of the nonlinear steady state response of the system compared to time-domain methods by several orders. Using HBM, the non-linear displacement $q(t)$ can be expressed as:

$$
q(t)=\tilde{Q}_{0}+\sum_{i=1}^{n_{h}}\left(\tilde{Q}_{i}^{c} \cos m_{i} \omega t+\tilde{Q}_{i}^{s} \sin m_{i} \omega t\right)
$$

Where $\tilde{Q}_{i}^{c, s}$ are cosine and sine harmonic coefficients for $i^{\text {th }}$ harmonic; $\omega$ is the principal excitation frequency; $\tilde{Q}_{0}$ is the zero harmonic response; $n_{h}$ is the number of harmonics. Using such a approximation, the size of original unknown vector would be then expanded by $2 n_{h}+1$ times. The framework of HBM usually includes three components: non-linear solver, Alternating Frequency 


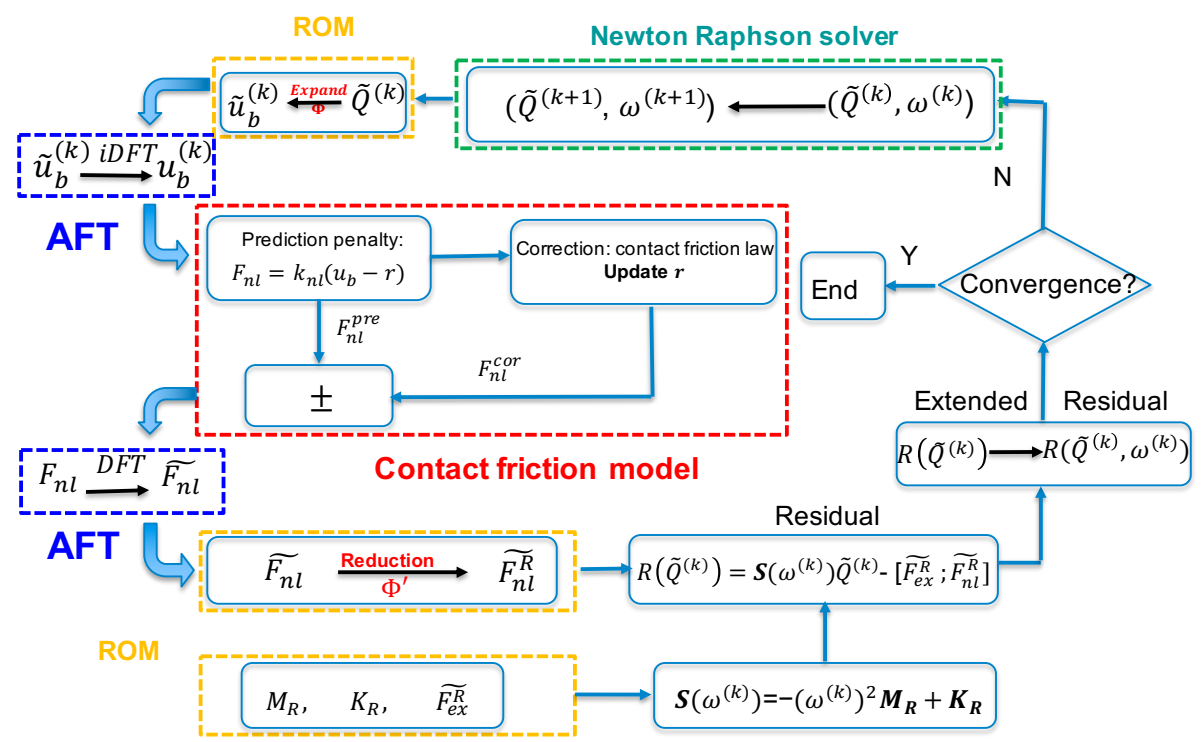

Figure 3: the flowchart of Correction procedure

Time (AFT) procedure and continuation technique [10. The Newton-Raphson method is employed as the non-linear iterative solver in this study. AFT techniques are used to transform the dynamic response and force between frequency and time domain in order to evaluate the contact friction force [10, 12. The continuation techniques 10, 24, namely using a predictor-corrector procedure, is used to to track the dynamic response with a particular parameter such that it can completely capture any soften or harden behaviour in the forced frequency response.

Fig 3 describes the key integration of the general ROM techniques into the classical HBM framework during the iterative correction. $k$ is denoted as the number of iterations. The flowchart starts from a predicted solution from the predictor, followed by a modal expansion procedure in order to obtain physical interface displacement on the interface, namely $\tilde{u}_{b}$ from the reduced unknown $\tilde{Q}^{(k)}$. The iDFT (Inverse Discrete Fourier Transform), as a part of AFT procedure, is then performed to transform the frequency domain $\tilde{u}_{b}$ into time domain $u_{b}$. The non-linear force $F_{n l}$ can be then calculated using the contact friction 


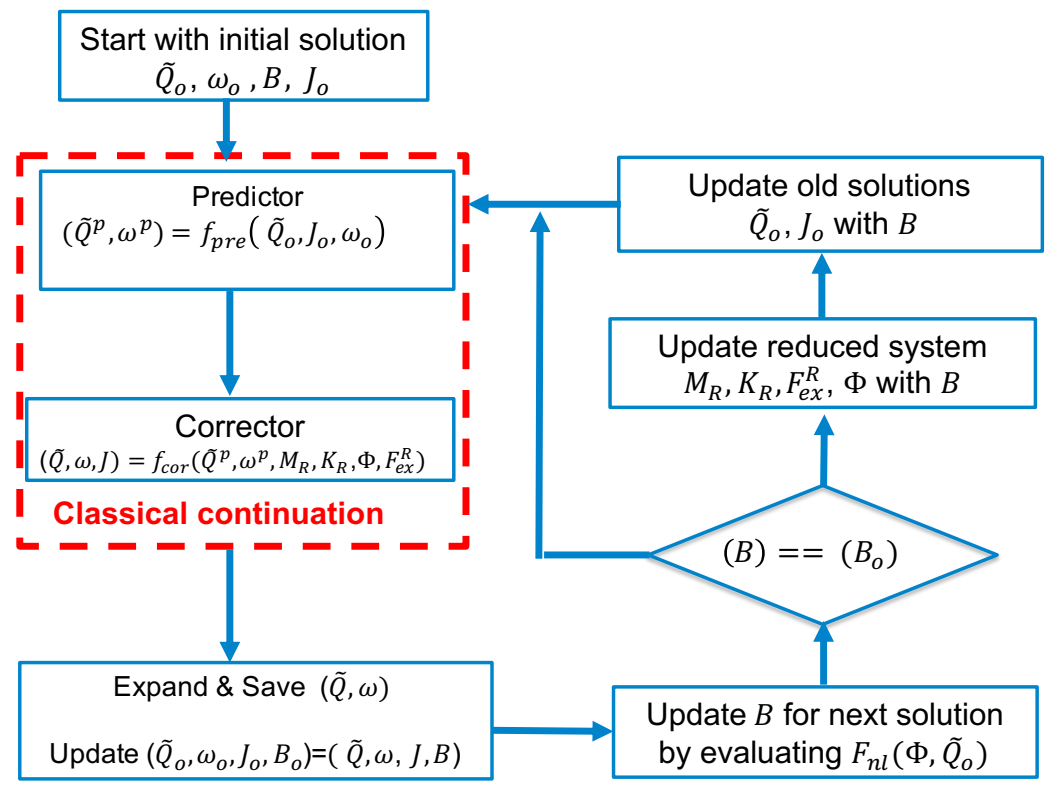

Figure 4: Algorithm describing the update of the problem size with varying contact conditions

model, and then projected back in modal subspace as $\tilde{F}_{n l}$ using DFT (Discrete Fourier Transform). The residual of the reduced dynamic system is then evaluated, which would be further extended by a correction constraint (e.g. Arclength) for the tracking parameter (excitation frequency in this study). If not convergent, the non-linear solver would iterate further $(k+1$ literation $)$ until a converged solution is found. Otherwise, the current solution is considered as the converged solution, which will be used in the predictor to estimate the next solution at a new frequency. In this way, the forced frequency response of the non-linear reduced system can be obtained. The full solution can be expanded from the reduced solution using $\Phi$.

\subsection{Automatic size updating algorithm}

In order to achieve automatic size update for the proposed adaptive ROM using harmonic balanced method, the challenge consists in two aspects:

- How to run continuous simulations for a reduced system with varying model size as contact conditions would change at the different frequencies? 
- How to predict the contact conditions accurately and efficiently based on the current solution for the simulation at the next frequency point?

Fig 4 describes the proposed algorithm to achieve the automatic update of the frequency analysis. The main feature consists in that the reduced system is at most updated once at each frequency before the continuation is performed. The updated reduced reduced model is determined based on the estimation of contact conditions from the last convergent solution. The detailed procedure of this algorithm is described in the following steps:

1. The simulation starts with the initial converged solution $\left(\tilde{Q}_{o}, \omega_{o}\right)$ together with Jacobean matrix $J_{o}$ and the index matrix $\mathbf{B}_{\mathbf{o}}$ that used to describe the contact conditions of interface nodes in Eq 19, It is followed a standard continuation procedure that includes a prediction stage to get a predicted solution $\left(\tilde{Q}^{p}, \omega^{p}\right)$ and a iterative correction stage to obtained the converged solution $(\tilde{Q}, \omega)$ (shown in Fig 3 . It is worth noting that the size of reduced system would remain consistent during the predictor and corrector procedure. Also, the contact force are only evaluated for those predicted slipping or gapping nodes and the other would be assumed in a stick condition.

2. Once a converged solution is obtained at a new frequency $\omega$, this solution needs to be expanded and saved in a full reduced size including all the zero components in $\Delta p$. This is to make sure all the solutions are saved in the same dimension that would be easy for post-procession. The old solution including $\left(\tilde{Q}_{o}, \omega_{o}, B_{o}, J_{o}\right)$ are also updated for the analysis at the next frequency.

3. The index matrix $\mathbf{B}$ for the next solution at a new frequency needs to be estimated before moving to the classical continuation procedure. It is because the contact condition for interface nodes can be different due to the change of excitation frequency. In this algorithm, the estimation 
of contact condition is performed by calling the non-linear contact force function using the last converged solution $\tilde{Q}_{o}$. The contact condition for every contact node will be evaluated in order to sufficiently realize the evolution of the slipping and gapping area on the contact interface.

4. If the new $\mathbf{B}$ is not the same as the previous $\mathbf{B}_{\mathbf{o}}$, it means the contact conditions have been changed from the last estimation. In this case, the reduced system $\mathbf{M}_{\mathbf{R}}, \mathbf{K}_{\mathbf{R}}, \boldsymbol{\Phi}, F_{e x}^{R}$ needs to be updated according to new B. Also, the size of last converged solution $\tilde{Q}_{o}, J_{o}$ need to be updated in order to make the system size consistent. After that, the simulation would continue to the classical continuation procedure.

5. If the new $\mathbf{B}$ turns out exactly same as the last $\mathbf{B}_{\mathbf{o}}$, it means the the contact conditions on the interface remain same. In this case, there is no need to update the system and solution for the next simulation. The process will directly jump to the classical continuation procedure at a continuously new frequency.

The proposed automatic updating algorithm is based on an assumption that it is reasonable to estimate the contact conditions at next excitation frequency from the last converged solution. This assumption can be justified by the fact that the slipping or gapping regions evolves continuously and slowly with the increase of excitation frequency. Once the sliding or gapping occurs, the system become non-linear and the step of frequency change is reduced thanks to the continuation technique, the converged solution therefore can be easily reached. In this algorithm, the reduced system needs only updated at most for the simulation at each frequency. It can significantly reduce the computational cost because the process of estimating the contact conditions and updating the reduced system can be very time-consuming if they are included in the iterative correction. The other major advantage of this implementation is that the classical continuation part in HBM keeps unchanged during the process of automatic size updating. It makes the proposed method well compatible with the existing HBM framework, and thus easy to be integrated. 


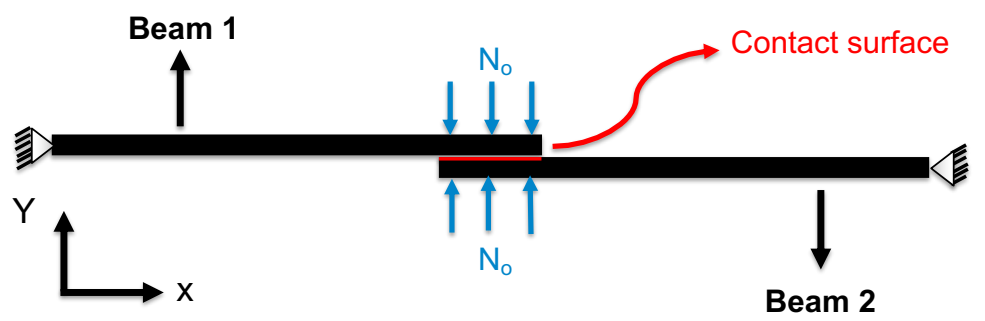

Figure 5: A 2D joint beam model

Most importantly, with the proposed method, huge computational cost spent in correction stage can be saved. It is mainly because the size of reduced system only retain the interface DOFs in a slipping or separating condition that would be much less than the classical CMS based reduced model. The other reason is, instead of calculation the contact friction for all the interface nodes, the calculation is only performed to those estimated slipping or gapping nodes.

\section{Case study: 2D joint beam}

\subsection{Finite element model}

Fig 5 shows a jointed beam model with linear springs connecting the two equivalent beam substructures. The length of each beam is $300 \mathrm{~mm}$. The width and height of the cross section is $25 \mathrm{~mm}$ and $6 \mathrm{~mm}$ respectively. They are modelled by using the Euler-Bernoulli beam elements, where each node has three DOFs $\left(u_{x}, u_{y}, r_{z}\right)$. The beams are made of steel with a nominal density of $7850 \mathrm{~kg} / \mathrm{m} 3$ and Young's modulus of $2.1 \mathrm{e} 11 \mathrm{~N} . \mathrm{m}^{-2} .250$ Bernoulli beam elements are used for each beam. The contact interface modelled by Jenkin elements involves 50 contact pairs, namely 300 contact friction DOFs. The total DOFs of FE model is 1500 . For the Jenkin element, the tangential and normal contact stiffness $k_{t}, k_{n}$ is $1 e 3 N / m$. Uniform distribution is used for the pre-loading $N_{o}$ on the contact friction interface.

Fig 6 shows the first nine modes of the $2 \mathrm{D}$ linearised jointed beam system assuming all the contact nodes are in a sticking condition. It shows the bending 

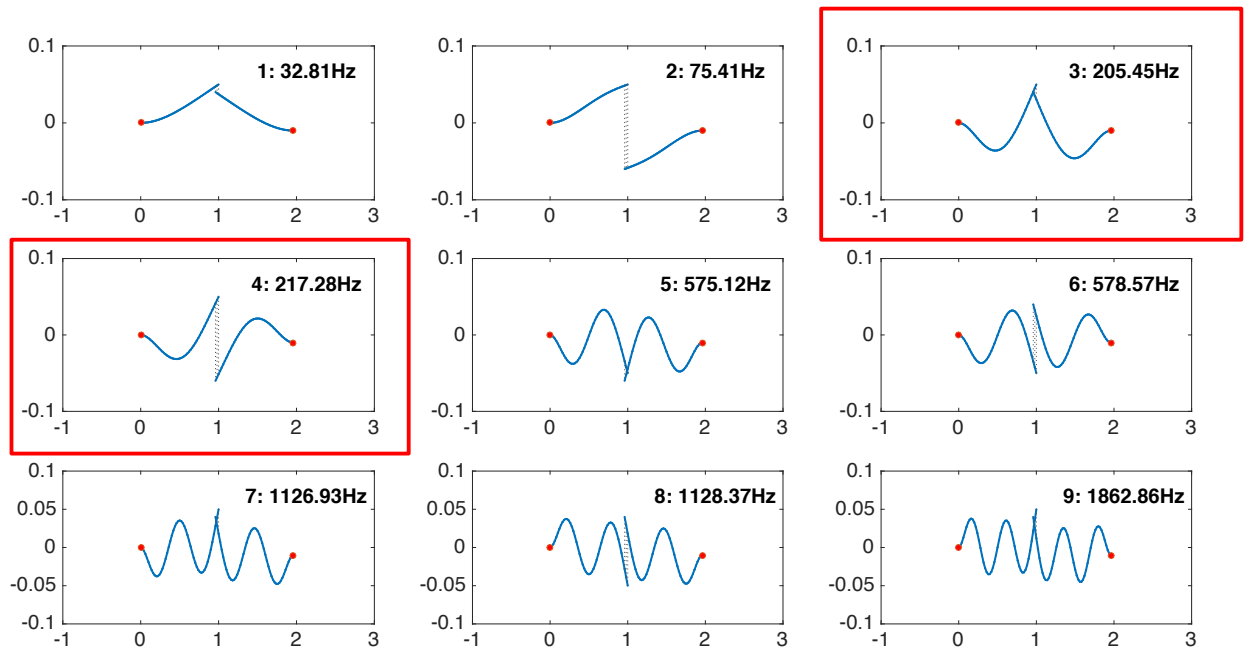

Figure 6: The mode shapes of linearised 2D joint beam

motion domains the modes of the first 9 modes. For this study, the $2^{\text {nd }}$ inphase and out-of-phase bending mode would be studied in the forced frequency response analysis.

\subsection{Forced frequency response}

Fig 7 shows the FRFs of the 2D jointed beam at different excitation levels. The structure is under a harmonic excitation in the middle of the upper beam in normal direction. The first peak refers to in phase second bending mode while the second peak refers to the out-of-phase one. With the increase of excitation levels, the gapping gradually occures on the contact interface making the resonance frequency of both modes shift left side, which is called softening effects. It is clearly that the out-of-phase mode are more significantly influenced than the in-phase mode due to the fact that the amplitude of out-of-phase motion is larger than in-phase one.

Fig 8 shows the contact condition of 50 contact pairs on the jointed interface at each frequency point. To be noted, the number of frequency points rather than the frequency itself is used as the horizontal axis in Fig. 8 , 9 and also 


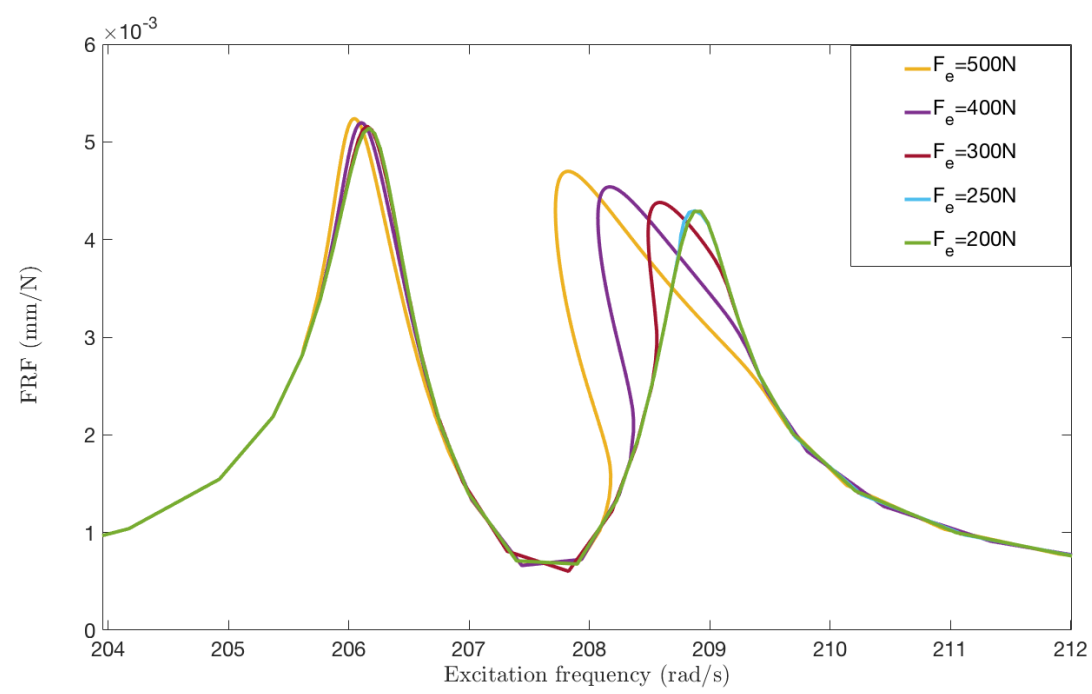

Figure 7: Forced frequency response of 2D jointed beam at different excitation levels

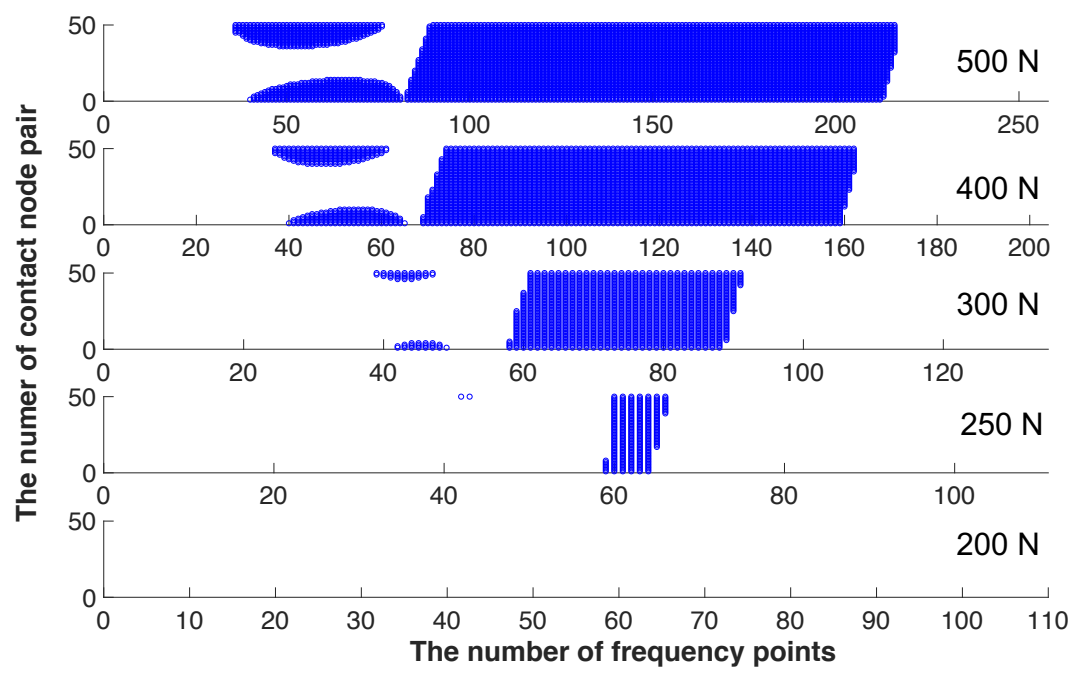

Figure 8: Contact conditions of the nodes in the joint interface 


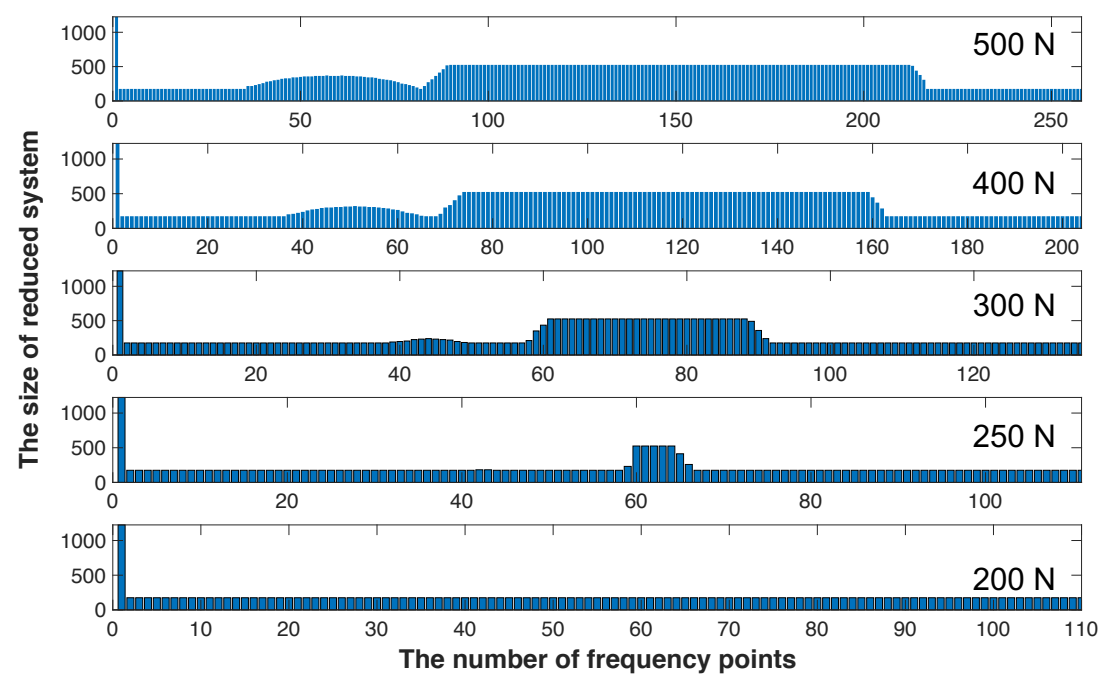

Figure 9: Automatic updated size of the system during the continuation

in Fig 14, 16. It is because that the corresponding forced response point for

a frequency close to the resonance might not be unique due to the soften or harden effects. For example, in Fig 7, at the frequency of $208 \mathrm{~Hz}$, there are 3 corresponding forced response points due to the soften effect. As expected, in this $2 \mathrm{D}$ case, there is no slipping state on the contact nodes because the normal and tangential motion is decoupled. The blue point means the gapping occurs on the contact interface. The area of the gapping zone expands gradually with the increase of excitation force level $F_{o}$. For the in-phase mode, the gapping propagates slowly from both edges of the interface toward the centre part of the interface. For the out-of-phase mode, the gapping expands quickly from one edge to the other and the joint interface becomes completely open at large excitation levels.

Fig 9 shows the size of a reduced system using the proposed method with automatic updating algorithm. The size of system can be effectively updated at each frequency point according to the contact conditions in Fig 8 At the excitation level of $200 \mathrm{~N}$, the DOFs of reduced system reaches minimum 30, which 


\begin{tabular}{|c|c|c|c|c|c|c|c|}
\hline & \multicolumn{3}{|c|}{ With auto update } & \multicolumn{3}{c|}{ Without auto update } & Speedup \\
\hline Force Level & Time & Evaluations & Points & Time & Evaluations & Points & Time per Evaluation \\
\hline $\mathrm{F}=500 \mathrm{~N}$ & $71.3 \mathrm{~s}$ & 1078 & 258 & $226.0 \mathrm{~s}$ & 1078 & 256 & $\mathbf{3 . 1 7}$ \\
\hline $\mathrm{F}=400 \mathrm{~N}$ & $55.9 \mathrm{~s}$ & 950 & 213 & $196.0 \mathrm{~s}$ & 929 & 212 & $\mathbf{3 . 5 1}$ \\
\hline $\mathrm{F}=300 \mathrm{~N}$ & $26.1 \mathrm{~s}$ & 646 & 154 & $136.3 \mathrm{~s}$ & 651 & 154 & $\mathbf{5 . 2 2}$ \\
\hline $\mathrm{F}=250 \mathrm{~N}$ & $16.2 \mathrm{~s}$ & 583 & 138 & $126.8 \mathrm{~s}$ & 622 & 139 & $\mathbf{7 . 8 3}$ \\
\hline $\mathrm{F}=200 \mathrm{~N}$ & $9.9 \mathrm{~s}$ & 543 & 135 & $120.8 \mathrm{~s}$ & 543 & 135 & $\mathbf{1 2 . 2 0}$ \\
\hline
\end{tabular}

Table 1: Comparison of the computational cost between the proposed method with and without automatic update

is the one sixth of the original reduced system. The size of system increases in proportionally with the number of gapping DOFs at a higher excitation level. Even in the case when the interface is completely open (e.g. at the excitation level of $500 \mathrm{~N}$ ), the method can also reduce the size of the system to one third of the size in original reduced system. It is because the tangential DOFs of contact nodes are still in a sticking condition, which are not added the reduced model. Without the automatic size updating, the reduced system would remain constantly at 180 DOFs including 150 DOFs on the joint interface and 30 modal DOFs. Table 1 shows the comparison of computational time of the proposed method with and without automatic update. The automatic update is able to improve the speedup factor (time per evaluation) by up to 12.20 . The range of the speedup factor depends on the contact conditions of the contact nodes on the interface.

\subsection{Accuracy and computational cost}

Rubin method is used to benchmark the results from the proposed method.

365 The amount of total DOFs for the Rubin ROM is 330, which includes 15 free interface vibrational modes and 150 static modes from the contact interface for each beam. Fig 10 shows the comparison of FRFs between Rubin and proposed method at different excitation levels. It shows that the proposed method is accurate enough to capture the forced frequency response at all excitation levels. Table 2 shows the comparison of computational cost between the proposed 


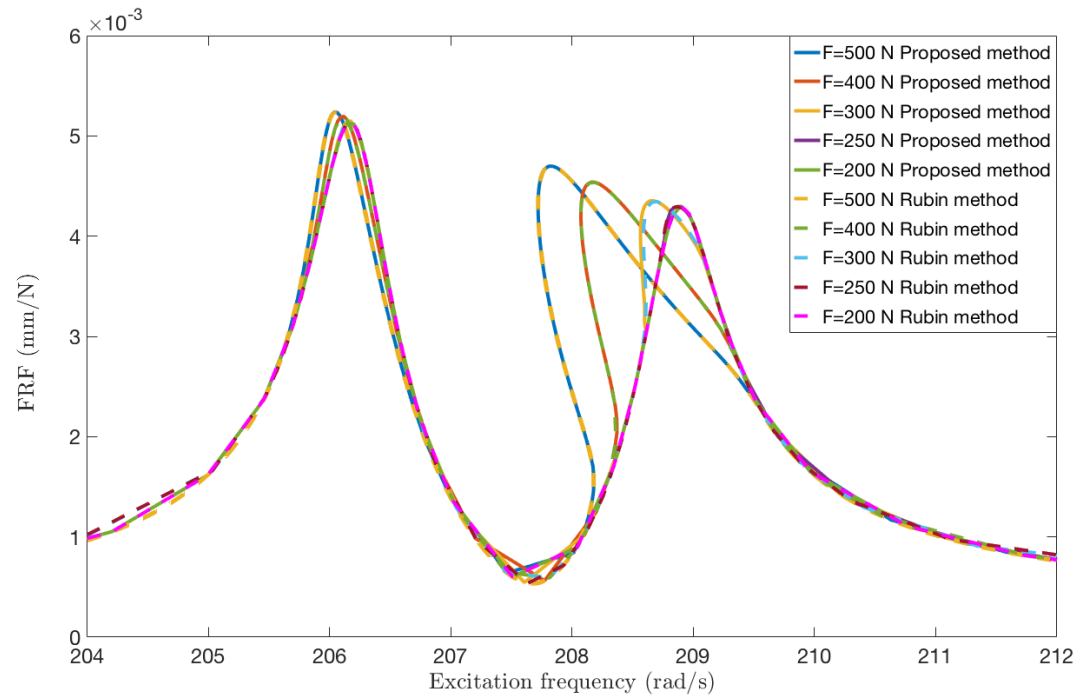

Figure 10: The comparison of the FRFs in 2D joint beam with Rubin method at different excitation levels

method and Rubin method. As expected, the computing speedup of the proposed method is proportional to the updating size of reduced system shown in Fig.9. The proposed method with auto size updating can achieve the speedup between 10.10 and 35.77 comparing to classic Rubin method. This is almost 3 times higher than the speedup achieved with the proposed method without updating algorithm at the same excitation level. This is due to that the Rubin method like all other CMS based reduction method remains all the interface DOFs in a ROM. One can also observe that the propose method can increase the speedup factor by 10 even when contact interface is completely open at a 380 excitation frequency close to the resonance frequency of the out-of-phase mode. This indicates that the proposed method with automatic size updating can also achieve a significant speedup in the macro-slip or macro-gapping motion. 


\begin{tabular}{|c|c|c|c|c|c|c|c|}
\hline & \multicolumn{3}{|c|}{ Proposed method } & \multicolumn{3}{c|}{ Rubin method } & Speedup \\
\hline Force Level & Time & Evaluations & Points & Time & Evaluations & Points & Time per Evaluation \\
\hline $\mathrm{F}=500 \mathrm{~N}$ & $71.3 \mathrm{~s}$ & 1078 & 258 & $297.4 \mathrm{~s}$ & 445 & 79 & $\mathbf{1 0 . 1 0}$ \\
\hline $\mathrm{F}=400 \mathrm{~N}$ & $55.9 \mathrm{~s}$ & 950 & 213 & $301.5 \mathrm{~s}$ & 460 & 82 & $\mathbf{1 1 . 1 4}$ \\
\hline $\mathrm{F}=300 \mathrm{~N}$ & $26.1 \mathrm{~s}$ & 646 & 154 & $310.3 \mathrm{~s}$ & 487 & 87 & $\mathbf{1 5 . 7 7}$ \\
\hline $\mathrm{F}=250 \mathrm{~N}$ & $16.2 \mathrm{~s}$ & 583 & 138 & $295.2 \mathrm{~s}$ & 467 & 85 & $\mathbf{2 2 . 7 5}$ \\
\hline $\mathrm{F}=200 \mathrm{~N}$ & $9.9 \mathrm{~s}$ & 543 & 135 & $315.0 \mathrm{~s}$ & 483 & 89 & $\mathbf{3 5 . 7 7}$ \\
\hline
\end{tabular}

Table 2: Comparison of the computational cost between the proposed method and Rubin method

\section{Case study: 3D joint beam}

\subsection{Finite element model}

Fig 11 shows a 3D finite element model of joint beam. The size of the upper beam is $40 x 20 x 5 \mathrm{~mm}$ while the lower beam is $140 x 20 x 5 \mathrm{~mm}$. The size of square contact interface between the 3D beams is $20 x 20 \mathrm{~mm}$. The joint structure is meshed by C3D8 solid element. The total DOFs of FE model is 9009 including 726 contact friction DOFs, namely 121 contact pairs on the joint interface. Titanium is used as the material for the joint beam, with the Young's modulus of $116 G P a$, density of $4.506 \mathrm{~g} / \mathrm{cm}^{3}$ and Poisson ratio of 0.32 . In terms of boundary condition, the left hand side of the upper beam is clamped while the other side with the bottom beam is left free. The uniform pressure distribution $N_{O}$ is applied to the contact interface. The normal and tangential contact stiffness $K_{t}$ and $K_{n}$ is chosen as $10^{3} \mathrm{~N} / \mathrm{mm}$. 0.3 is selected as the friction coefficient $\mu$.

Figure 12 shows the first four vibrational modes of the 3D joint beam when the contact interface is linearised with the contact stiffness. The first and third mode are the first two in-plane bending modes. The second one is the edgewise flapping while the forth one is the torsional mode. In this study, the second edge-wise flapping mode would be studied for forced frequency response analysis. 


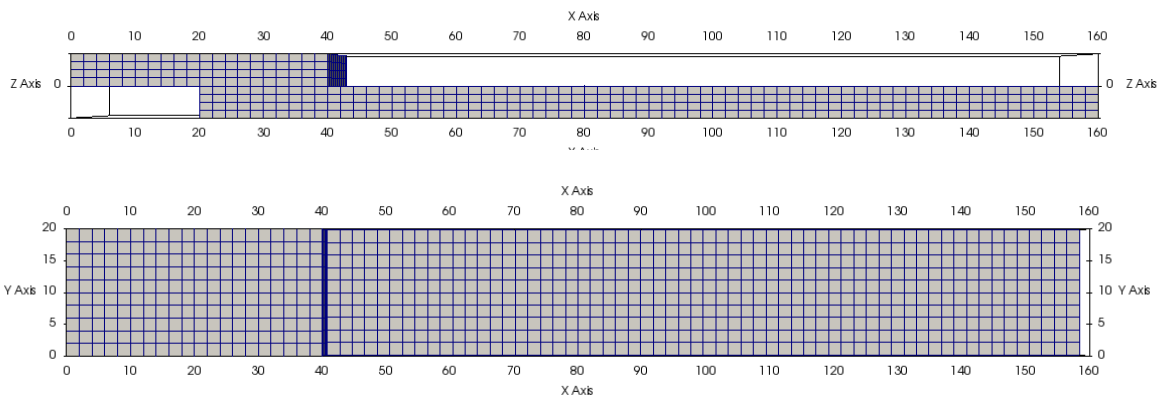

Figure 11: A finite element model of 3D joint beam

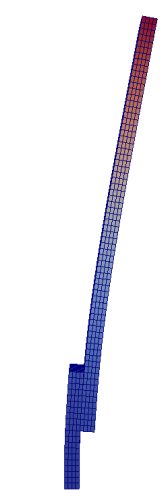

Mode1

First in-plane flapping $1010 \mathrm{~Hz}$

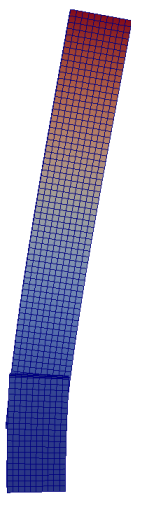

Mode2

First edge-wise flapping $3260 \mathrm{~Hz}$

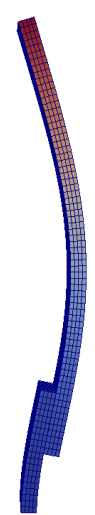

Mode3

Second in-plane flapping $6060 \mathrm{~Hz}$

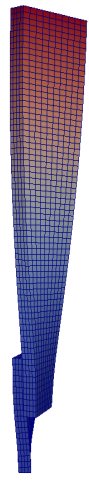

Mode4

First Torsion $13950 \mathrm{~Hz}$

Figure 12: Mode shapes of the 3D joint beam 


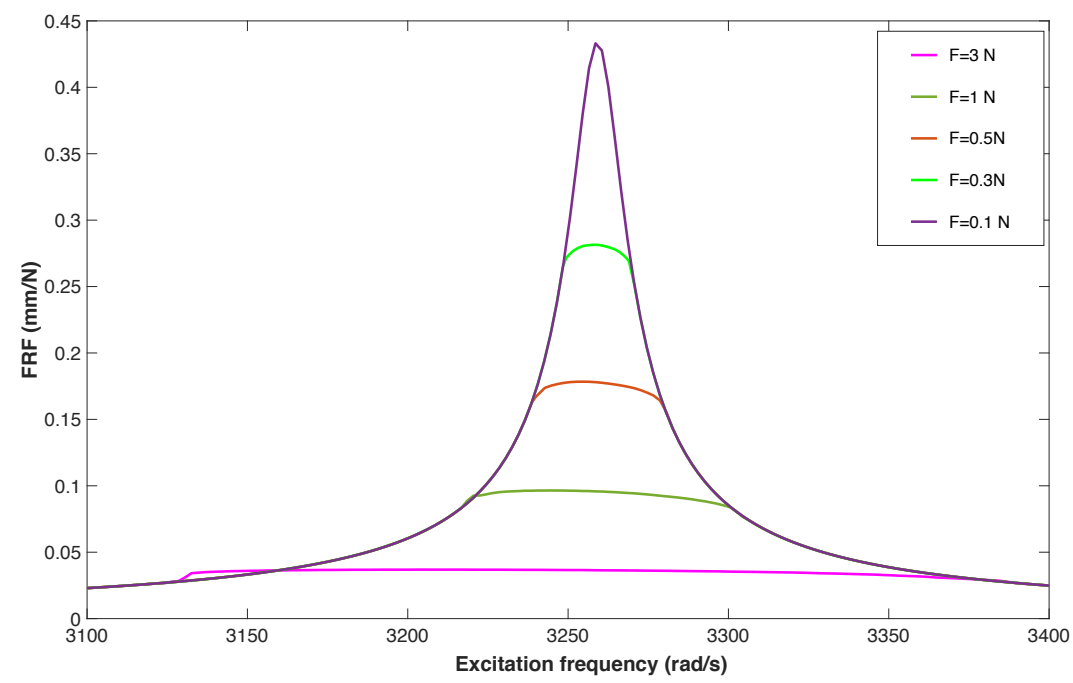

Figure 13: Forced frequency response of 3D jointed beam at different excitation levels

\subsection{Forced frequency response}

The jointed structure is forced by the harmonic excitation in the $Y$ direction in the middle of the free beam. Fig 13 shows the forced frequency response function (FRF) of the joint structure at different excitation levels. 3 harmonics are used in HBM. With the increase of the excitation level, the resonance peak of the second mode decreases significantly and become flat due to the energy dissipated by friction forces on the interface.

Fig 14 show the contact condition of 121 contact pairs in the tangential direction at each frequency point, which corresponds to FRF curve in Fig 13 . In this case, gapping does not occur in the normal direction for all the contact nodes. The area of micro-slip in tangential directions expands with the excitation levels. At the excitation level of $3 \mathrm{~N}$, the slipping region propagates to the most of the contact interface. Fig $[15]$ shows the distribution of the slipping nodes in the contact interface at different excitation levels at the corresponding resonance frequencies. The blue dot indicates the slipping occurs at that contact node. It shows the slipping starts from the edges of the contact interface 

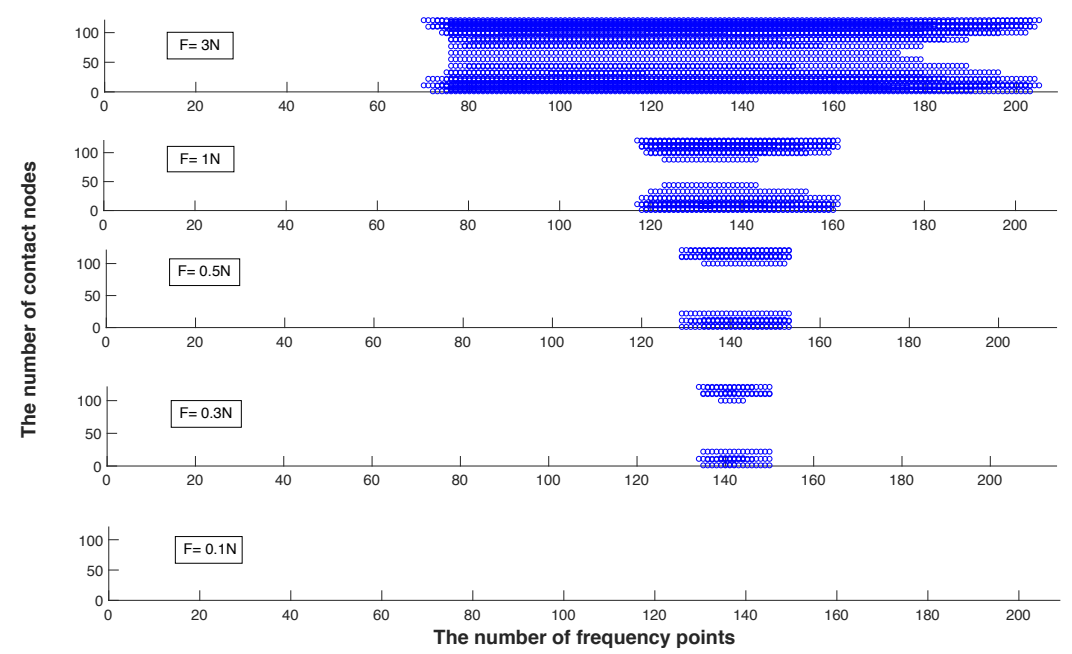

Figure 14: Contact condition of the interface in tangential direction

and then gradually propagates to the centre with the increase of the excitation levels.

Fig 16 shows the updating size of the reduced system using the proposed method with automatic size updating at excitation level of $3 \mathrm{~N}, 1 \mathrm{~N}, 0.5 \mathrm{~N}$ and $0.1 \mathrm{~N}$. As expected, the size of reduced system are effectively updated according to the contact condition of the interface shown in Fig 13 At the excitation of $0.1 \mathrm{~N}$, the size of the system keeps at minimum value of 350 for all the frequency points because the whole interface is in a sticking condition. The size of reduced system gradually increases and expands close to the resonance area at a higher excitation level. Even at the excitation level of $3 \mathrm{~N}$ (most of contact nodes are in a slipping condition), the size of system can be reduce to less than one third of orginal size. Table 3 shows the comparison of computational time between the proposed method with and without automatic size updating. It shows the proposed method coupling with the automatic size updating can effectively improve computational time by a speedup between 12.83 to 26.68 . Obviously, the maximum speedup factor is achieved at a lowest excitation level. This further validates the effectiveness of the proposed automatic updating algorithm. 


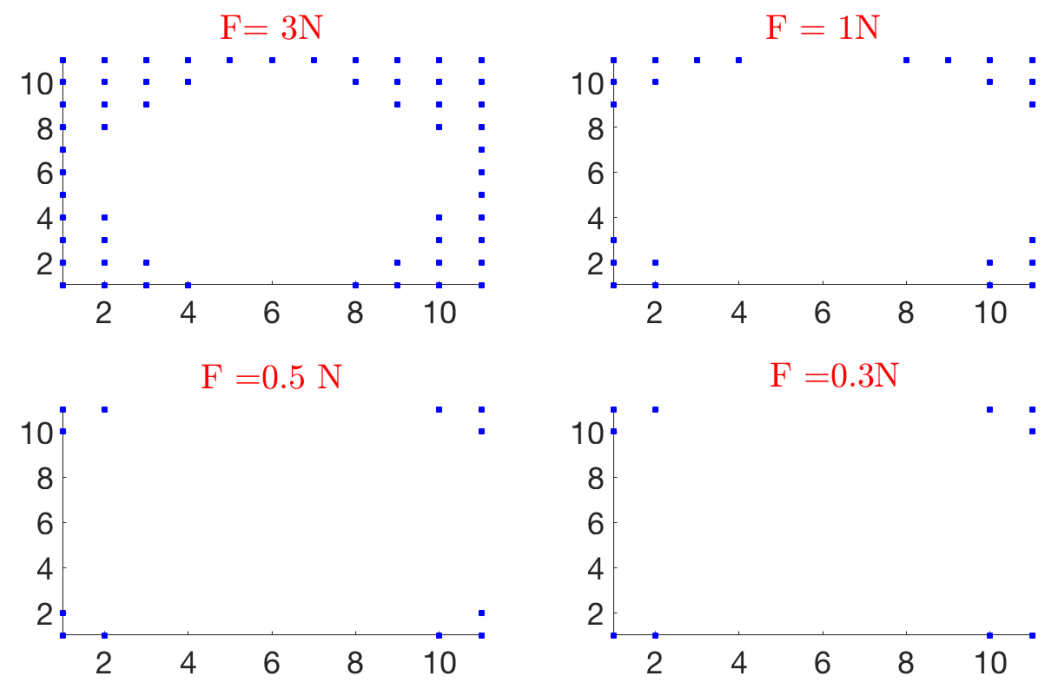

Figure 15: The distribution of slipping contact nodes in joint interface

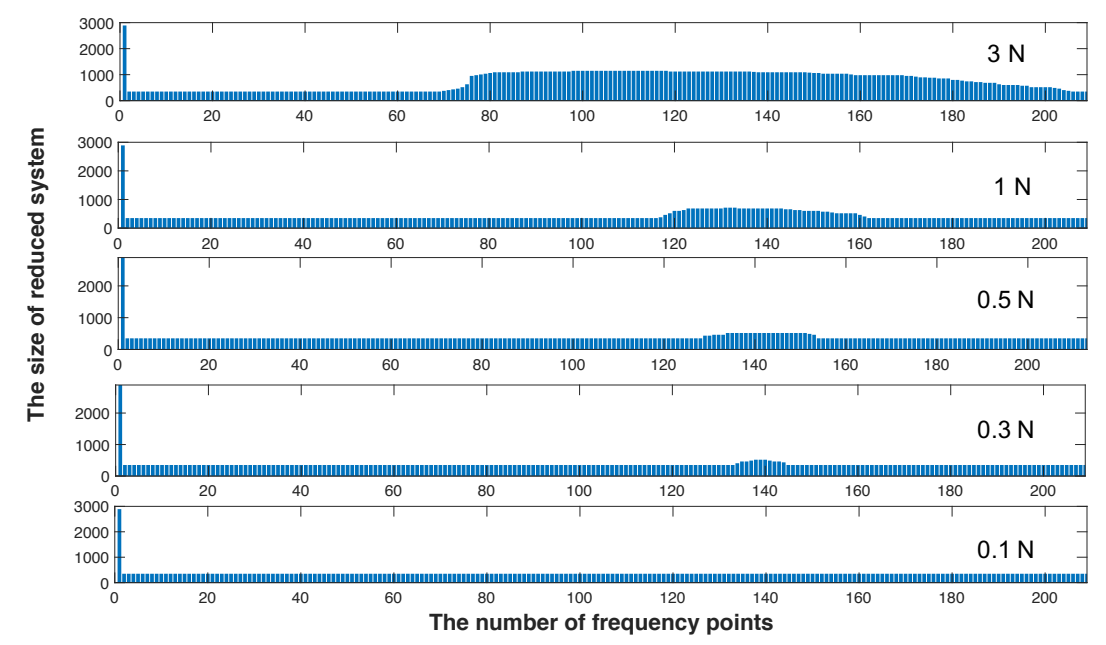

Figure 16: The automatic updating size of the system during the continuation 


\begin{tabular}{|c|c|c|c|c|c|c|c|}
\hline & \multicolumn{3}{|c|}{ With auto update } & \multicolumn{3}{c|}{ Without auto update } & Speedup \\
\hline Force Level & Time & Evaluations & Points & Time & Evaluations & Points & Time per Evaluation \\
\hline F $=3 \mathrm{~N}$ & $554.5 \mathrm{~s}$ & 1332 & 209 & $3547.5 \mathrm{~s}$ & 664 & 209 & $\mathbf{1 2 . 8 3}$ \\
\hline $\mathrm{F}=1 \mathrm{~N}$ & $162.2 \mathrm{~s}$ & 679 & 209 & $3633.2 \mathrm{~s}$ & 672 & 209 & $\mathbf{2 2 . 6 3}$ \\
\hline $\mathrm{F}=0.5 \mathrm{~N}$ & $149.7 \mathrm{~s}$ & 718 & 213 & $3693.5 \mathrm{~s}$ & 683 & 209 & $\mathbf{2 5 . 9 4}$ \\
\hline $\mathrm{F}=0.3 \mathrm{~N}$ & $143.6 \mathrm{~s}$ & 706 & 209 & $3858.5 \mathrm{~s}$ & 711 & 211 & $\mathbf{2 6 . 6 8}$ \\
\hline $\mathrm{F}=0.1 \mathrm{~N}$ & $138.5 \mathrm{~s}$ & 664 & 209 & $3572.7 \mathrm{~s}$ & 643 & 209 & $\mathbf{2 6 . 6 8}$ \\
\hline
\end{tabular}

Table 3: Comparison of the computational cost between the proposed method with and without automatic update

\begin{tabular}{|c|c|c|c|c|c|c|c|}
\hline & \multicolumn{3}{|c|}{ Proposed method } & \multicolumn{3}{c|}{ Rubin method } & Speedup \\
\hline Force Level & Time & Evaluations & Points & Time & Evaluations & Points & Time per Evaluation \\
\hline F $=3 \mathrm{~N}$ & $554.5 \mathrm{~s}$ & 1332 & 209 & $7790.7 \mathrm{~s}$ & 424 & 58 & $\mathbf{4 4 . 1 4}$ \\
\hline $\mathrm{F}=1 \mathrm{~N}$ & $162.2 \mathrm{~s}$ & 679 & 209 & $3946.2 \mathrm{~s}$ & 223 & 37 & $\mathbf{7 4 . 0 8}$ \\
\hline $\mathrm{F}=0.5 \mathrm{~N}$ & $149.7 \mathrm{~s}$ & 718 & 213 & $5882.3 \mathrm{~s}$ & 309 & 54 & $\mathbf{9 1 . 3 0}$ \\
\hline $\mathrm{F}=0.3 \mathrm{~N}$ & $143.6 \mathrm{~s}$ & 706 & 209 & $7264.4 \mathrm{~s}$ & 391 & 72 & $\mathbf{9 1 . 3 3}$ \\
\hline $\mathrm{F}=0.1 \mathrm{~N}$ & $138.5 \mathrm{~s}$ & 664 & 209 & $6970.5 \mathrm{~s}$ & 362 & 363 & $\mathbf{9 2 . 3 2}$ \\
\hline
\end{tabular}

Table 4: Comparison of the computational cost between the proposed method and Rubin method

\subsection{Accuracy and computational cost}

Fig 17 shows a comparison of the FRFs between the proposed method and Rubin method at the same four excitation levels. It shows the proposed method is able to capture the dynamic response of this $3 \mathrm{D}$ joint beams at all four excitation levels. Table 4 shows the comparison of the computational cost of the proposed method and Rubin method. It shows that the proposed method can achieve a speedup factor between 44 and 92 . The obtained speedup factors are about 2.5 times higher than that achieved in 2D test case shown in Table 2

This means the speed-up achieved by the proposed method not only depends on the contact conditions on the interface but also depends on the size of contact interface in FE model. The larger size of contact region, the bigger speedup can be possibly achieved. For 3D test case, the number of contact pairs is 121 which is 2.4 time higher than the contact pair number of $2 \mathrm{D}$ case 50 , which is is similar to the times of the increased speedup. 


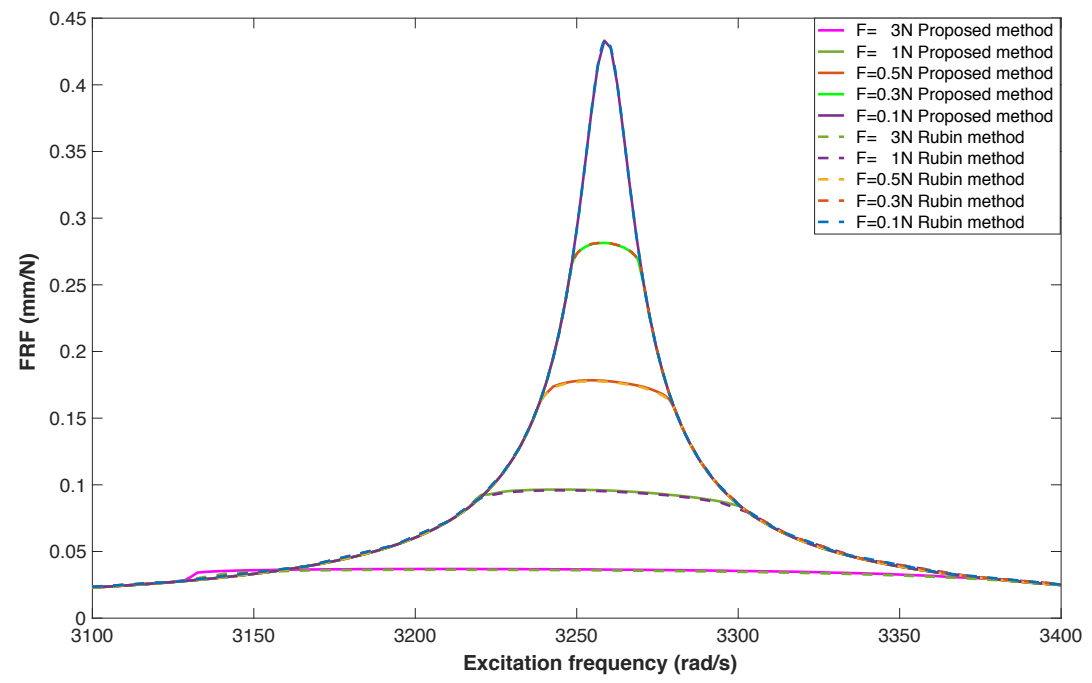

Figure 17: The comparison of the FRF with Hybrid method

\section{Conclusions}

A novel adaptive ROM technique for dynamic analysis of the jointed structure with contact friction interfaces has been presented. The main feature of the proposed method is that the reduced model can be automatically update its size according to the contact condition of contact interface nodes, which is able to significantly reduce the size of state of the art CMS based reduced models. The idea of the proposed ROM method mainly consists in a new formulation of dynamic system that combines a linearised joint system with an internal penalty variable that accounts non-linear effects introduced by the slipping or separation phenomenons on the joint interfaces. This makes the resulting reduced order model able to only includes the contact DOFs in a slip or gap condition in its

reduced basis. A enormous reduction can be achieved for the jointed structure as most of their interface nodes are in a stick condition for jointed structures. To achieve the automatic size updating with the non-linear solver, a new algorithm to update its model size according to the varying contact condition has been presented. The proposed algorithm is well compatible with classic continuation 

the contact condition for the next solution at a continuous excitation frequency.

The proposed method has been tested in two distinct case studies, one with a $2 \mathrm{D}$ jointed beam and the other with a $3 \mathrm{D}$ joint beam. As expected, for both case studies, the proposed method is able to automatically update size of reduced system according to the contact conditions of nodes on the joint interface. For the forced response analysis, a significant speedup has been achieved for both studies at different excitation levels. The maximum computing speedup, 36 for the $2 \mathrm{D}$ case and 92 for the $3 \mathrm{D}$ case, occurs when the whole contact interface is in a stick condition. However, even when the contact interface is mostly in a response at all the excitation levels for both case studies. This study proves that the proposed adaptive reduction technique can significantly improve the computational efficiency of CMS based reduction method for the prediction of dynamics of joint structures. The study also validates that the proposed auto495 slipping/gapping condition, the proposed method can still achieve speed up of 10 for the $2 \mathrm{D}$ case and 40 for the $3 \mathrm{D}$ case. This is mainly because that the size of reduced system is still comparably less than benchmarking Rubin method via removing the interface linear DOFs from the other directions, e.g. the normal contact DOFs can be still removed in a slip condition. Thanks to the proposed updating algorithm, the proposed ROM techniques works very well with popular harmonic balanced method for spectral analysis. The implementation of the updating algorithm is simple because the classical continuation part can remain unaffected.

The results also suggest that the efficiency of the proposed techniques depends not only on excitation levels but also on the size of contact friction interface. The larger the size of contact interface is, the bigger the computing speedup can be achieved. In this study, the speedup factor for $3 \mathrm{D}$ case is almost 3 times higher than that for $2 \mathrm{D}$ case. The speedup ratio is similar to the ratio of contact size between $3 \mathrm{D}$ and $2 \mathrm{D}$ case. With respect to the accuracy, the proposed ROM method is able to accurately capture the forced frequency matic size updating algorithm can work very well with HBM and the proposed 
ROM technique.

\section{Acknowledgement}

The authors would like to acknowledge the support of Rolls-Royce plc and Innovate UK through GEMiniDS WP3 -Innovate UK Project 113088.

[1] C. Schwingshackl, E. Petrov, D. Ewins, Measured and estimated friction interface parameters in a nonlinear dynamic analysis, Mechanical Systems and Signal Processing 28 (2012) 574-584.

[2] M. R. W. Brake, D. J. Ewins, C. B. Wynn, Are Joints Necessary?, Springer International Publishing, Cham, 2018, pp. 25-36.

[3] H. G. D. Goyder, Damping Due to Joints in Built-Up Structures, Springer International Publishing, Cham, 2018, pp. 135-147.

[4] B. Titurus, J. Yuan, F. Scarpa, S. Patsias, S. Pattison, Impact hammerbased analysis of nonlinear effects in bolted lap joint, in: Proceedings of the ISMA 2016 International Conference on Noise and Vibration Engineering, KU Leuven, Departement Werktuigkunde, 2016, pp. 789-801.

[5] R. Lacayo, L. Pesaresi, J. Groß, D. Fochler, J. Armand, L. Salles, C. Schwingshackl, M. Allen, M. Brake, Nonlinear modeling of structures with bolted joints: A comparison of two approaches based on a time-domain and frequency-domain solver, Mechanical Systems and Signal Processing 114 (2019) 413-438.

[6] S. Bograd, P. Reuss, A. Schmidt, L. Gaul, M. Mayer, Modeling the dynamics of mechanical joints, Mechanical Systems and Signal Processing 25 (8) (2011) 2801-2826. 

M. J. Starr, B. R. Resor, Handbook on dynamics of jointed structures., Tech. rep., Sandia National Laboratories (2009).

[8] S. Huang, Dynamic analysis of assembled structures with nonlinearity, Ph.D. thesis, Dynamics group, Department of Mechanical Engineering, Imperial College London (2008).

[9] E. Petrov, A high-accuracy model reduction for analysis of nonlinear vibrations in structures with contact interfaces, Journal of Engineering for Gas Turbines and Power 133 (10) (2011) 102503.

[10] M. Krack, L. Salles, F. Thouverez, Vibration prediction of bladed disks coupled by friction joints, Archives of Computational Methods in Engineering 24 (3) (2017) 589-636.

[11] M. R. W. Brake, J. Groß, R. M. Lacayo, L. Salles, C. W. Schwingshackl, P. Reuß, J. Armand, Reduced Order Modeling of Nonlinear Structures with Frictional Interfaces, Springer International Publishing, Cham, 2018, pp. $427-450$.

[12] J. Yuan, F. El-Haddad, L. Salles, C. Wong, Numerical assessment of reduced order modeling techniques for dynamic analysis of jointed structures with contact nonlinearities, Journal of Engineering for Gas Turbines and Power 141 (3) (2019) 031027.

[13] G. Battiato, C. Firrone, T. Berruti, B. Epureanu, Reduction and coupling of substructures via gram-schmidt interface modes, Computer Methods in Applied Mechanics and Engineering 336 (2018) 187-212.

[14] J. Yuan, F. Scarpa, G. Allegri, B. Titurus, S. Patsias, R. Rajasekaran, Efficient computational techniques for mistuning analysis of bladed discs: A review, Mechanical Systems and Signal Processing 87 (2017) 71-90. 
[15] R. Pinnau, Model reduction via proper orthogonal decomposition, in: Model Order Reduction: Theory, Research Aspects and Applications, Springer, 2008, pp. 95-109.

[16] S. Zucca, B. I. Epureanu, Reduced order models for nonlinear dynamic analysis of structures with intermittent contacts, Journal of Vibration and Control 24 (12) (2018) 2591-2604.

[17] S. Rubin, Improved component-mode representation for structural dynamic analysis, AIAA journal 13 (8) (1975) 995-1006.

[18] R. Craig, M. Bampton, Coupling of substructures for dynamic analyses, AIAA journal 6 (7) (1968) 1313-1319.

[19] W. Witteveen, H. Irschik, Efficient mode based computational approach for jointed structures: joint interface modes, AIAA Journal 47 (1) (2009) $252-263$.

[20] J. Becker, L. Gaul, Cms methods for efficient damping prediction for structures with friction, Proceedings of the IMAC-XXVI, Orlando.

[21] L. Gaul, J. Becker, Damping prediction of structures with bolted joints, Shock and Vibration 17 (4-5) (2010) 359-371.

[22] J. Yuan, L. Salles, C. Wong, S. Patsias, A novel penalty-based reduced order modelling method for dynamic analysis of joint structures, in: IUTAM Symposium on Model Order Reduction of Coupled Systems, Stuttgart, Germany, May 22-25, 2018.

[23] F. M. Gruber, D. J. Rixen, Evaluation of substructure reduction techniques with fixed and free interfaces, Strojniški vestnik-Journal of Mechanical Engineering 62 (7-8) (2016) 452-462.

[24] E. Sarrouy, J.-J. Sinou, Non-linear periodic and quasi-periodic vibrations in mechanical systems-on the use of the harmonic balance methods, in: Advances in Vibration Analysis Research, InTech, 2011. 